\title{
Non-compact Complex Projective Superspaces by Hamiltonian reduction
}

\section{Erik Khastyan ${ }^{a, *}$}

${ }^{a}$ Yerevan Physics Institute,

2 Alikhanian Brothers St., Yerevan 0036, Armenia

E-mail: erik.khastyan@yerphi.am

We briefly describe some concepts of super-Hamiltonian reduction and complex projective spaces. Next, we are going to perform super-Hamiltonian reduction in a concrete case, namely to get compact and non-compact complex projective superspaces from complex (pseudo)Euclidean superspaces. Then we concentrate on the non-compact case, and specifically on the supergeneralization of $N$-dimensional Klein model and the mapping of superconformal mechanics on it.

RDP online workshop "Recent Advances in Mathematical Physics" - Regio2020,

5-6 December 2020

online

${ }^{*}$ Speaker 


\section{Introduction}

Kähler spaces and specifically projective spaces are very interesting from the physical point of view. Since they are maximally symmetric spaces it is natural to consider physical systems on them. Moreover, due to their symplectic structure it is also natural to consider them as a phase spaces. Let us maintain that systems with Kähler phase spaces can be quantized in an elegant way via geometric quantization. An integrable system with Kähler phase space was suggested in [4] and is called compactified Ruijsenaars-Schneider system (relativistic Calogero model). Also there exists an explicit mapping of the phase space of this system to the complex projective space which was done in [5]. As we have maintained above, it can be quantized using geometric quantization methods $[6,7]$.

In the series of papers ([8-11]) so called Calogero-Oscillator and Calogero-Coulomb systems had been studied in spherical coordinates and by the use of compex coordinates an intresting results were obtained. $N$-extended superconformal extension of these systems can be constructed for one-dimensional case [12]. In [2] is shown how are complex coordinates and Kähler phase space related. In that paper authors proposed the description of superintegrable models with dynamical so(1.2) symmetry, and of the generic superintegrable deformations of oscillator and Coulomb systems in terms of higher-dimensional Klein model playing the role of phase space. Saying higher-dimensional Klein model we mean the non-compact analog of the complex projective space. The key point was that it was possible to express the constants of motion of those systems via Killing potentials defining the $s u(N .1)$ isometries of the Kähler structure.

In our belief, these techniques that were established ([2]) for the pure bosonic case can be very usefully aplied to the study of supersimmetrizations as well. Some preliminary paper ([13]) was published devoted to the compact and non-compact complex projective spaces that can be obtained from complex (pseudo)Euclidean spaces by Hamiltonian reduction. In this paper we are going to perform a similar steps for the, so called, compact and non-compact Complex projective superspaces using super-Hamiltonian reduction. To have an idea about super-Hamiltonian reduction and related topics see [1]. In [3] the supergeneralization of $C P^{N}$ (compact complex projective space) provided with even and odd Kähler structures are constructed using Hamiltonian reduction. Let us make some remarks about super-Hamiltonian reduction. Roughly speaking the super-Hamiltonian formalism is a straightforward generalization of ordinary Hamiltonian formalism plus sign factors that are related with Grassmann parity. From supergeometrically point of view the only perceptible difference is the existence of the odd Poisson brackets (anti-brackets) which has no analogs in the ordinary theory.

The Poisson brackets of the functions $f(x)$ and $g(x)$ on superspaces are defined by the expression

$$
\{f, g\}_{\kappa}=\frac{\partial_{r} f}{\partial x^{A}} \Omega_{\kappa}^{A B}(x) \frac{\partial_{l} g}{\partial x^{B}}, \quad \kappa=0,1 .
$$

They obey the conditions

$$
\begin{aligned}
& p\left(\{f, g\}_{\kappa}\right)=p(f)+p(g)+\kappa \quad \text { (grading), } \\
& \{f, g\}_{\kappa}=-(-1)^{(p(f)+\kappa)(p(g)+\kappa)}\{g, f\}_{\kappa} \quad \text { ("antisymmetricity"), } \\
& (-1)^{(p(f)+1)(p(h)+\kappa)}\left\{f,\{g, h\}_{\kappa}\right\}_{\kappa}+c . p .=0 \quad \text { (Jacobi identity). }
\end{aligned}
$$


Here $x^{A}$ are local coordinates of superspace, while $\partial_{r} / \partial x^{A}$ and $\partial_{l} / \partial x^{A}$ denote right and left derivatives respectively.

Clearly the nondegenerate odd Poisson brackets can be defined on the $(N . N)$-dimensional superspaces, and the nondegenerate even Poisson brackets could be defined on (2N.M)-dimensional ones. In this case the Poisson brackets are associated with the supersymmetric structure

$$
\Omega_{\kappa}=d z^{A} \Omega_{(\kappa) A B} d z^{B}, \quad d \Omega_{\kappa}=0,
$$

where $\Omega_{(\kappa) A B} \Omega_{\kappa}^{B C}=\delta_{A}^{C}$.

In the further discussion in this paper only even brackets $(\kappa=0)$ will be discussed. In this paper we are going to perform super-Hamiltonian reduction in a concrete case, especially to get compact and non-compact complex projective spaces from complex (pseudo)Euclidean spaces. And then we will concentrate on the non-compact case, and specifically on the supergeneralized $N$-dimensional Klein model, which will be explained later, and the mapping of superconformal mechanics on it.

\section{Compact and non-compact Complex Projective Superspaces}

The compact and non-compact complex projective superspaces $C P^{N \mid M}$ and $\widetilde{C P}^{N \mid M}$ can be obtained by super-Hamiltonian reduction of Complex Euclidean $C^{N+1 \mid M}$ and Complex pseudoEuclidean $C^{N .1 \mid M}$ superspaces.

Let us consider the complex superspace $C^{N+1 \mid M}\left(C^{N .1 \mid M}\right)$ parameterized by the complex coordinates $\left(u^{a}, \eta^{A}\right)$, where $a=0,1, \ldots, N$ and $A=1, \ldots, M$. Let us equip it with the canonical symplectic structure

$$
\Omega_{0}=\imath\left(d u^{0} \wedge d \bar{u}^{0} \pm \sum_{a=1}^{N} d u^{a} \wedge d \bar{u}^{a}+\imath \sum_{A=1}^{M} d \eta^{A} \wedge d \bar{\eta}^{A}\right) .
$$

and associated Poisson brackets

$$
\begin{aligned}
& \left\{u^{a}, \bar{u}^{b}\right\}=\imath \delta^{a \bar{b}}, \quad \text { (compact) } \\
& \left\{u^{a}, \bar{u}^{b}\right\}=\imath \gamma^{a \bar{b}}, \quad(\text { non }- \text { compact })
\end{aligned} \quad\left\{\eta^{A}, \bar{\eta}^{B}\right\}=\left\{\bar{\eta}^{B}, \eta^{A}\right\}=\delta^{A \bar{B}},
$$

where $a, b=0,1, \ldots, N$ and $\gamma=\operatorname{diag}(1,-1, \cdots,-1)$.

On this superspace we can define the linear Hamiltonian action of $u(N .1 \mid M)=u(1) \times$ $s u(N .1 \mid M)$ superalgebra

$$
\begin{gathered}
\left\{h_{a \bar{b}}, h_{c \bar{d}}\right\}=\imath\left(h_{a \bar{d}} \delta^{b \bar{c}}-h_{c \bar{b}} \delta^{d \bar{a}}\right), \quad\left\{R_{A \bar{B}}, R_{C \bar{D}}\right\}=\imath\left(R_{A \bar{D}} \delta^{B \bar{C}}-R_{C \bar{B}} \delta^{D \bar{A}}\right), \\
\left\{\Theta_{A \bar{a}}, \Theta_{\bar{B} b}\right\}=h_{b \bar{a}} \delta^{B \bar{A}}-R_{A \bar{B}} \delta^{a \bar{b}} \\
\left\{\Theta_{A \bar{a}}, h_{b \bar{c}}\right\}=\imath \Theta_{A \bar{c}} \delta^{a \bar{b}}, \quad\left\{\Theta_{A \bar{a}}, R_{B \bar{C}}\right\}=-\imath \Theta_{B \bar{a}} \delta^{C \bar{A}}
\end{gathered}
$$

where

$$
h_{a \bar{b}}=\bar{u}^{a} u^{b}, \quad \Theta_{A \bar{a}}=\bar{\eta}^{A} u^{a}, \quad R_{A \bar{B}}=\imath \bar{\eta}^{A} \eta^{B} .
$$


The $u(1)$ generator defining the center of $u(N .1 \mid M)$ is given by the expression

$$
J=u^{0} \bar{u}^{0} \pm \sum_{a=1}^{N} u^{a} \bar{u}^{a}+i \sum_{A=1}^{M} \eta^{A} \bar{\eta}^{A}: \quad\left\{J, h_{a \bar{b}}\right\}=\left\{J, \Theta_{A \bar{a}}\right\}=\left\{J, R_{A \bar{B}}\right\}=0 .
$$

The reduction of $C^{N+1 \mid M}\left(C^{N .1 \mid M}\right)$ by this generator will bring as to (non-compact) complex projective superspace $C P^{N \mid M}\left(\widetilde{C P}^{N \mid M}\right)$. Since this reduction yields the $(2 N \mid 2 M)$-(real) dimensional phase space we have to choose, at first, the $2 N$ real ( $N$ complex) bosonic functions commuting with $J_{0}$, and $2 M$ real ( $M$ complex) fermionic ones. Then, we have to calculate their Poisson brackets and restrict the latter to the level surface

$$
J=g .
$$

As a result we will get the Poisson brackets on the reduced $(2 N \mid 2 M)$-(real) dimensional space, with that $U(1)$-invariant functions playing the role of the latter's coordinates:

Let us choose the following $U(1)$ invaiant functions

$$
z^{a}=\frac{u^{a}}{u^{0}}, \quad \theta^{A}=\frac{\eta^{A}}{u^{0}}: \quad\left\{z^{a}, J\right\}=\left\{\theta^{A}, J\right\}=0, \quad \text { and } \quad \text { c.c. : }
$$

notice that from now on the indices $a$ are running from 1 to $\mathrm{N}$. Then, from the equation (13) we get the expression

$$
A:=\left.\frac{1}{u^{0} \bar{u}^{0}}\right|_{J=g}=\frac{1 \pm \sum_{c} z^{c} \bar{z}^{c}+\imath \sum_{C} \theta^{C} \bar{\theta}^{C}}{g}
$$

So, calculating the Poisson brackets of the functions (14) and having in mind the above expression we arrive to the reduced Poisson brackets defined by the following non-zero relations (and their complex conjugates)

$$
\left\{z^{a}, \bar{z}^{b}\right\}=\frac{z^{a} \bar{z}^{b}+\delta^{a \bar{b}}}{A}, \quad\left\{z^{a}, \bar{\theta}^{A}\right\}=\frac{z^{a} \bar{\theta}^{A}}{A}, \quad\left\{\theta^{A}, \bar{\theta}^{B}\right\}=\frac{\theta^{A} \bar{\theta}^{B}+\delta^{A \bar{B}}}{A} .
$$

These Poisson brackets are defined by the following Kähler potential(s)

$$
\mathcal{K}= \pm g \log \left(1 \pm \sum_{c} z^{c} \bar{z}^{c}+\imath \sum_{C} \theta^{C} \bar{\theta}^{C}\right)
$$

Indeed, we have arrived to the Kähler potentials that are associated with $(N \mid M)$-dimensional compact complex projective superspace $C P^{N \mid M}$ (for the upper sign) and $(N \mid M)$-dimensional noncompact complex projective superspace $\widetilde{C P}^{N \mid M}$ (for the lower sign). From thes Kḧler potentials one can deduce an $s u(N+1 \mid M)$-invariant (for the compact case) and an $s u(N .1 \mid M)$ invariant (for the non-compact case) Kähler metrics, which are somehow the super generalizations of the Fubini-Study ones.

$$
d s^{2}=g \frac{d z d \bar{z} \pm \imath d \theta d \bar{\theta}}{\left(1 \pm z^{c} \bar{z}^{c}+\imath \theta^{C} \bar{\theta}^{C}\right)} \mp g \frac{(\bar{z} d z \pm \imath \bar{\theta} d \theta)(z d \bar{z} \mp \imath \theta d \bar{\theta})}{\left(1 \pm z^{c} \bar{z}^{c}+\imath \theta^{C} \bar{\theta}^{C}\right)^{2}}
$$

The isometry algebra of this space(s) is $s u(N+1 \mid M)(s u(N .1 \mid M))$. It is defined by the following Killing potentials

$$
h_{a \bar{b}}=\left.\bar{u}^{a} u^{b}\right|_{J=g}=\frac{\bar{z}^{a} z^{b}}{A}, \quad \Theta_{A \bar{a}}=\left.\bar{\eta}^{A} u^{a}\right|_{J=g}=\frac{\bar{\theta}^{A} z^{a}}{A}, \quad R_{A \bar{B}}=\left.\imath \bar{\eta}^{A} \eta^{B}\right|_{J=g}=\frac{\bar{\theta}^{A} \theta^{B}}{A} .
$$


where $A$ is defined in formula (15). One could write these generators in canonical coordinates using the following transformation,

$$
z^{a}=\sqrt{\frac{p_{a}}{g+p-\imath \chi^{C} \bar{\chi}^{C}}} e^{\imath \varphi_{a}}, \quad \theta^{A}=-\frac{\chi^{A}}{\sqrt{g+p-\imath \chi^{C} \bar{\chi}^{C}}}, \quad p=\sum_{a} p_{a},
$$

with

$$
\left\{\varphi_{a}, p_{b}\right\}=\delta_{a b}, \quad\left\{\chi^{A}, \bar{\chi}^{B}\right\}=\delta^{A \bar{B}}
$$

\section{A superextended Klein model}

To present the linear realization of $s u(N .1 \mid M)$ superconformal algebra let us consider the complex pseudo-Euclidian superspace $C^{N .1 \mid M}$ equipped with the canonical Kähler structure, and thus, by the canonical supersymplectic structure

$$
\Omega_{0}=\imath \sum_{a, b=0}^{N} \gamma_{a \bar{b}} d v^{a} \wedge d \bar{v}^{b}+\sum_{A=1}^{M} d \eta^{A} \wedge d \bar{\eta}^{A},
$$

with $v^{a}, \bar{v}^{a}$ being bosonic variables, and $\eta^{A}, \bar{\eta}^{A}$ being fermionic ones, and with the matrix $\gamma_{a \bar{b}}$ chosen in the form

$$
\gamma=\left(\begin{array}{cc|ccc}
0 & -i & & & \\
i & 0 & & & \\
\hline & & -1 & & \\
& & & \ddots & \\
& & & & -1
\end{array}\right), \quad a, b=0,1, \ldots, N-1 .
$$

For the transition to the diagonal metrics $\gamma=\operatorname{diag}(1,-1, \ldots,-1)$ one should simply perform the transformation

$$
v^{0} \rightarrow \frac{v^{0}+v^{N}}{\sqrt{2}}, \quad v^{N} \rightarrow \frac{v^{0}-v^{N}}{\imath \sqrt{2}}
$$

With this supersymplectic structure we can associate the Poisson brackets given by the relations

$$
\left\{v^{a}, \bar{v}^{b}\right\}=-l \gamma^{\bar{b} a}, \quad\left\{\eta^{A}, \bar{\eta}^{B}\right\}=\left\{\bar{\eta}^{B}, \eta^{A}\right\}=\delta^{A \bar{B}}, \quad \gamma^{\bar{a} b} \gamma_{b \bar{c}}=\delta_{c}^{a} .
$$

Equivalently,

$$
\left\{v^{0}, \bar{v}^{N}\right\}=1, \quad\left\{v^{N}, \bar{v}^{0}\right\}=-1, \quad\left\{v^{\alpha}, \bar{v}^{\beta}\right\}=\imath \delta^{\alpha \bar{\beta}}, \quad\left\{\eta^{A}, \bar{\eta}^{B}\right\}=\left\{\bar{\eta}^{B}, \eta^{A}\right\}=\delta^{A \bar{B}},
$$

here we introduced the indices $\alpha, \beta=1, \ldots, N-1$.

On this superspace we can define the linear Hamiltonian action of $u(N .1 \mid M)=u(1) \times$ $s u(N .1 \mid M)$ superalgebra

$$
\begin{gathered}
\left\{h_{a \bar{b}}, h_{c \bar{d}}\right\}=-\imath\left(h_{a \bar{d}} \gamma^{\bar{c} b}-h_{c \bar{b}} \gamma^{\bar{a} d}\right), \quad\left\{R_{A \bar{B}}, R_{C \bar{D}}\right\}=\imath\left(R_{A \bar{D}} \delta^{B \bar{C}}-R_{C \bar{B}} \delta^{D \bar{A}}\right), \\
\left\{\Theta_{A \bar{a}}, \Theta_{\bar{B} b}\right\}=h_{b \bar{a}} \delta^{B \bar{A}}-R_{A \bar{B}} \gamma^{\bar{b} a}, \\
\left\{\Theta_{A \bar{a}}, h_{b \bar{c}}\right\}=-\imath \Theta_{A \bar{c}} \gamma^{\bar{b} a}, \quad\left\{\Theta_{A \bar{a}}, R_{B \bar{C}}\right\}=-\imath \Theta_{B \bar{a}} \delta^{C \bar{A}},
\end{gathered}
$$


where

$$
h_{a \bar{b}}=\bar{v}^{a} v^{b}, \quad \Theta_{A \bar{a}}=\bar{\eta}^{A} v^{a}, \quad R_{A \bar{B}}=\imath \bar{\eta}^{A} \eta^{B} .
$$

The $u(1)$ generator defining the center of $u(N .1 \mid M)$ is given by the expression

$$
J=\gamma_{a \bar{b}} v^{a} \bar{v}^{b}+\imath \eta^{A} \bar{\eta}^{A}: \quad\left\{J, h_{a \bar{b}}\right\}=\left\{J, \Theta_{A \bar{a}}\right\}=\left\{J, R_{A \bar{B}}\right\}=0 .
$$

Hence, reducing the system by the action of this generator we will get the "non-compact" projective super-space $\widetilde{C P}^{N \mid M}$ (i.e. the supergeneralization of noncompact projective space $\widetilde{C P}^{N}$ ). Since this reduction yields the $(2 N \mid 2 M)$-(real) dimensional phase space we have to choose, at first, the $2 N$ real ( $N$ complex) bosonic functions commuting with $J_{0}$, and $2 M$ real ( $N$ complex) fermionic ones. Then, we have to calculate their Poisson brackets and restrict the latter to the level surface

$$
J=g .
$$

As a result we will get the Poisson brackets on the reduced $(2 N \mid 2 M)$-(real) dimensional space, with that $U(1)$-invariant functions playing the role of the latter's coordinates.

Let us choose the following $U(1)$ invaiant functions

$$
w=\frac{v^{N}}{v^{0}}, \quad z^{\alpha}=\frac{v^{\alpha}}{v^{0}}, \quad \theta^{A}=\frac{\eta^{A}}{v^{0}}: \quad\{w, J\}=\left\{z^{a}, J\right\}=\left\{\theta^{A}, J\right\}=0, \quad \text { and } \quad \text { c.c.. }
$$

Then, from the equation (32) we get the expression

$$
A:=\left.\frac{1}{v^{0} \bar{v}^{0}}\right|_{J=g}=\frac{\imath(w-\bar{w})-z^{\gamma} \bar{z}^{\gamma}+\imath \theta^{C} \bar{\theta}^{C}}{g}
$$

So, calculating the Poisson brackets of the functions (33) and having in mind the above expression we arrive to the reduced Poisson brackets defined by the following non-zero relations (and their complex conjugates)

$$
\begin{gathered}
\{w, \bar{w}\}=-A(w-\bar{w}), \quad\left\{z^{\alpha}, \bar{z}^{\beta}\right\}=\imath A \delta^{\alpha \bar{\beta}}, \quad\left\{\theta^{A}, \bar{\theta}^{B}\right\}=A \delta^{A \bar{B}}, \\
\left\{w, \bar{z}^{\alpha}\right\}=A \bar{z}^{\alpha}, \quad\left\{w, \bar{\theta}^{A}\right\}=A \bar{\theta}^{A} .
\end{gathered}
$$

These Poisson brackets are associated with the supersymplectic structure

$$
\begin{aligned}
\Omega=\frac{l}{g} & {\left[\frac{1}{A^{2}} d w \wedge d \bar{w}-\frac{l z^{\alpha}}{A^{2}} d w \wedge d \bar{z}^{\alpha}-\frac{\theta^{A}}{A^{2}} d w \wedge d \bar{\theta}^{A}\right.} \\
& +\frac{l \bar{z}^{\alpha}}{A^{2}} d z^{\alpha} \wedge d \bar{w}+\left(\frac{g \delta_{\alpha \bar{\beta}}}{A}+\frac{\bar{z}^{\alpha} z^{\beta}}{A^{2}}\right) d z^{\alpha} \wedge d \bar{z}^{\beta}-\frac{l \bar{z}^{\alpha} \theta^{A}}{A^{2}} d z^{\alpha} \wedge d \bar{\theta}^{A} \\
& \left.-\frac{\bar{\theta}^{A}}{A^{2}} d \theta^{A} \wedge d \bar{w}+\frac{l \bar{\theta}^{A} z^{\alpha}}{A^{2}} d \theta^{A} \wedge d \bar{z}^{\alpha}-\left(\frac{\lg \delta_{A \bar{B}}}{A}+\frac{\bar{\theta}^{A} \theta^{B}}{A^{2}}\right) d \theta^{A} \wedge d \bar{\theta}^{B}\right] .
\end{aligned}
$$

It is defined by the Kähler potential

$$
\mathcal{K}=-g \log \left(\imath(w-\bar{w})-z^{\alpha} \bar{z}^{\alpha}+\imath \theta^{A} \bar{\theta}^{A}\right) .
$$

Now, let us perform the following transformation

$$
w \rightarrow l \frac{z^{N}-1}{z^{N}+1}, \quad z^{\alpha} \rightarrow \sqrt{2} \frac{z^{\alpha}}{z^{N}+1}, \quad \theta^{A} \rightarrow \sqrt{2} \frac{\theta^{A}}{z^{N}+1},
$$


which is basically the same as (24). Doing so we will get the Kähler potential

$$
\mathcal{K}=-g \log \left(1-z^{c} \bar{z}^{c}+\imath \theta^{C} \bar{\theta}^{C}\right) .
$$

In what follows we will call this space "noncompact projective superspace $\widetilde{C P}^{N \mid M}$ ". The isometry algebra of this space is $s u(N .1 \mid M)$, which can be easily obtained by the restriction of (29) to the level surface (32). It is defined by the following Killing potentials

$$
\begin{gathered}
H:=\left.v^{N} \bar{v}^{N}\right|_{J=g}=\frac{w \bar{w}}{A}, \quad K:=\left.v^{0} \bar{v}^{0}\right|_{J=g}=\frac{1}{A}, \quad D:=\left.\left(v^{N} \bar{v}^{0}+v^{0} \bar{v}^{N}\right)\right|_{J=g}=\frac{w+\bar{w}}{A}, \\
H_{\alpha}:=\left.\bar{v}^{\alpha} v^{N}\right|_{J=g}=\frac{\bar{z}^{\alpha} w}{A}, \quad K_{\alpha}:=\left.\bar{v}^{\alpha} v^{0}\right|_{J=g}=\frac{\bar{z}^{\alpha}}{A}, \quad h_{\alpha \bar{\beta}}:=\left.\bar{v}^{\alpha} v^{\beta}\right|_{J=g}=\frac{\bar{z}^{\alpha} z^{\beta}}{A}, \\
Q_{A}:=\left.\bar{\eta}^{A} v^{N}\right|_{J=g}=\frac{\bar{\theta}^{A} w}{A}, \quad S_{A}:=\left.\bar{\eta}^{A} v^{0}\right|_{J=g}=\frac{\bar{\theta}^{A}}{A}, \quad \Theta_{A \bar{\alpha}}:=\left.\bar{\eta}^{A} v^{\alpha}\right|_{J=g}=\frac{\bar{\theta}^{A} z^{\alpha}}{A}, \\
R_{A \bar{B}}:=\left.\imath \bar{\eta}^{A} \eta^{B}\right|_{J=g}=l \frac{\bar{\theta}^{A} \theta^{B}}{A} .
\end{gathered}
$$

With these expressions of Killing potentials at hands we can construct a variety of superconformal mechanical systems defined by them.

The crucial thing here is that, just as in pure bosonic case, the isometries of Kähler space (Killing potentials) became isometries of the corresponding Hamiltonian system. Namely linear combinations presented in (41) correspond to the superconformal generators of the system, while expressions in (42)-(44) correspond to the additional symmetry generators (bosonic and fermionic).

One can write these generators in canonical coordinates using the following transformations

$$
w=\frac{p_{r}}{r}-\imath \frac{g+\sum_{\alpha=1}^{N-1} \pi_{\alpha}-\sum_{A=1}^{M} l \chi^{A} \bar{\chi}^{A}}{r^{2}}, \quad z^{\alpha}=\frac{\sqrt{2 \pi_{\alpha}}}{r} \mathrm{e}^{i \varphi_{\alpha}}, \quad \theta^{A}=-\frac{\sqrt{2}}{r} \chi^{A} .
$$

where

$$
\left\{r, p_{r}\right\}=1, \quad\left\{\varphi_{\beta}, \pi_{\alpha}\right\}=\delta_{\alpha \beta}, \quad\left\{\chi^{A}, \bar{\chi}^{B}\right\}=\delta^{A \bar{B}}, \quad \pi_{a} \geq 0, \quad \varphi^{a} \in[0,2 \pi), \quad r>0 .
$$

The relation with the canonical coordinates in (20) is as follows

$$
p_{N}=\frac{p_{r}^{2}}{4}+\frac{1}{4}\left(r-\frac{\sqrt{2 \mathcal{I}}}{r}\right)^{2}, \quad p_{\alpha}=\pi_{\alpha}, \quad \chi^{A}=\chi^{A} .
$$

In our next coming paper [14] we are going to give a several examples of $s u(1 . N \mid M)$ superconformal systems with Euclidian configuration spaces, that can be formulated in the framework considered above. As well as we are going to define and study the $2 M$-extended supersymmetric maximally superintegrable oscillator-like and Coulomb-like systems.

\section{Acknowledgments}

First and foremost I would like to acknowledge professor Armen Nersessian, whose invaluable support made this work possible. As well as, I acknowledge a partial financial support of Armenian Science Committee (grant 20RF-023) and of the Regional Doctoral Program on Theoretical and Experimental Particle Physics sponsored by VolkswagenStiftung and Armenian Committee of Science. This work was fulfilled within the ICTP Affiliated Center Program AF-04 and ICTP Network project NT-04. 


\section{References}

[1] A.Nersessian Elements of (super-)Hamiltonian Formalism Lect.Notes Phys.698 (2006) 139188

[2] Erik Khastyan, Armen Nersessian, Hovhannes Shmavonyan, Noncompact $C P^{N}$ as a phase space of superintegrable systems, [arXiv:2003.10002 [math-ph]] to appear in Int. J. Mod. Phys. A, 36 (2021),

[3] O. M. Khudaverdian, A. P. Nersessian, Even and odd symplectic and Kählerian structures on projective superspaces J. Math. Phys. 34 (1993) 5533.

[4] S. N. M. Ruijsenaars and H. Schneider, A New Class of Integrable Systems and Its Relation to Solitons, Annals Phys. 170 (1986) 370.

[5] S.N.M. Ruijsenaars, Action-angle maps and scattering theory for some finitedimensional integrable systems. III. Sutherland type systems and their duals. Publ.Res.Inst.Math.Sci.Kyoto,31(1995), 247-353

[6] J. F. van Diejen, L. Vinet, The Quantum Dynamics of the Compactified Trigonometric Ruijsenaars-Schneider Model, Commun. Math. Phys. 197(1998), 33-74 [arXiv:math/9709221[math-ph]]

[7] L. Feher and T. F. Görbe, Trigonometric and elliptic Ruijsenaars-Schneider systems on the complex projective space, Lett. Math. Phys. 106 (2016) no.10, 1429 [arXiv:1605.09736 [math$\mathrm{ph}]]$.

[8] T. Hakobyan, A. Nersessian, H. Shmavonyan Lobachevsky geometry in TTW and PW systems Phys.Atom.Nucl. 80 (2017) no.3, 598-604

[9] T. Hakobyan, A. Nersessian, H. Shmavonyan Constants of motion in deformed oscillator and Coulomb systems Phys.Part.Nucl.Lett. 14 (2017) no.2, 400-402

[10] T. Hakobyan, A. Nersessian, H. Shmavonyan Symmetries in superintegrable deformations of oscillator and Coulomb systems: Holomorphic factorization Phys.Rev. D95 (2017) no.2, 025014

[11] T. Hakobyan, O. Lechtenfeld and A. Nersessian, Superintegrability of generalized Calogero models with oscillator or Coulomb potential, Phys. Rev. D 90 (2014) no.10, 101701 [arXiv: 1409.8288 [hep-th]].

[12] T. Hakobyan and A. Nersessian, Lobachevsky geometry of (super)conformal mechanics, Phys. Lett. A 373 (2009) 1001 doi:10.1016/j.physleta.2009.01.036 [arXiv:0803.1293 [hep-th]].

[13] E. Khastyan, H. Shmavonyan Non-Compact Complex Projective Space as a Phase Space, Phys. Part. Nuclei Lett. 17, 744-747 (2020).

[14] E. Khastyan, A.Nersessian, Kähler geometry for su(N.1|M)-superconformal mechanics, in preparation 